Morales Valero, M., Resa Navarro, X. y Salas Velasco, M. (2021). La política evaluativa de formación profesional en España. Revista de Investigación Educativa, 39(2), 587-606.

DOI: http://dx.doi.org/10.6018/rie.454311

\title{
La política evaluativa de formación profesional en España
}

\section{The assessment policy of vocational training in spain}

\author{
Manuel Morales Valero*, Xavier Resa Navarro** y Manuel Salas Velasco***। \\ *Universidad de Málaga \\ ** Universitat Politècnica de Catalunya \\ ***Universidad de Granada
}

\begin{abstract}
Resumen
Presentamos un estudio de producción educativa de carácter exploratorio en el contexto del sistema de formación profesional de grado superior español. Entendemos como outputs educativos las finalidades propias de la formación profesional consistente en responder a las demandas del mercado laboral y lograr una adecuada inserción sociolaboral de sus egresados. El objetivo es doble. Por un lado, analizamos la influencia de determinados inputs "no escolares» como la «personalidad eficaz» y la «clase social subjetiva». Por otro, comprobamos el grado de congruencia existente entre los distintos modelos evaluativos utilizados en los dos ámbitos en que se desarrollan actualmente estas enseñanzas: el educativo y el laboral. Para ello, utilizamos una muestra no probabilística por conveniencia de 374 estudiantes pertenecientes al segundo curso de distintos ciclos formativos de grado superior situados en centros educativos de la provincia de Barcelona (España). Se les administró un cuestionario informatizado donde se recogían las principales variables explicativas tomadas en consideración. El análisis se basó en un modelo econométrico logit ordenado. Los resultados obtenidos señalan como la «expectativa de éxito», en tanto dimensión del constructo «personalidad eficaz», y la «clase social subjetiva» determinan positivamente los resultados. En segundo lugar, podemos afirmar que no existe relación alguna entre los resultados medidos por las evaluaciones realizadas desde el ámbito educativo y laboral. De este modo, a falta de una mayor reflexión
\end{abstract}

Correspondencia: Manuel Morales Valero, mmoralesvalero@uma.es, Bulevar Louis Pasteur, 25, D-2.46, 29010 Málaga. 
y evidencia científica, es posible que haya que replantear la politica evaluativa del sistema de formación profesional dando un paso más hacia su necesaria integración.

Palabras clave: formación profesional; criterio de evaluación; política de la educación; administración educativa.

\begin{abstract}
We present an exploratory educational production study in the context of the Spanish higher vocational training system. We consider educational outputs to be the specific purposes of professional training, i.e., responding to the demands of the labour market and achieving adequate social and labour integration for graduates. This article pursues two aims. On the one hand, we analyse the influence of certain "non-school" inputs such as "effective personality" and "subjective social class". On the other hand, we check the degree of congruence among the different assessment models used in the two areas in which these teachings are currently developed: education and labour. To this end, we used a non-probabilistic convenience sample of 374 second-year students from different higher degree training cycles located in educational centres in the province of Barcelona, Spain. The students were administered a computerised questionnaire which included the main explanatory variables taken into consideration. The analysis was based on an ordered econometric logit model. The results obtained indicate that the "expectation of success", as a dimension of the "effective personality" construct, and the "subjective social class" determine the results positively. Furthermore, we can state that there is no relationship among the results measured by the assessments carried out in the areas of education and labour. Thus, in the absence of greater reflection and scientific evidence, the assessment policy of the vocational training system may have to be reconsidered, taking a further step towards a needed integration.
\end{abstract}

Keywords: vocational training; assessment criterion; educational policy; educational administration.

\title{
Introducción
}

El presente estudio analiza los resultados del proceso productivo de educación superior postobligatoria en el ámbito de la formación profesional inicial para los ciclos formativos de grado superior del sistema educativo español. Por un lado, pretende determinar la influencia de los constructos «personalidad eficaz» y «clase social subjetiva» en tanto inputs "no escolares", y por otro, comprobar el grado de congruencia existente entre los indicadores de resultados (outputs) utilizados por los modelos evaluativos propios de los dos ámbitos en que se desarrolla: el educativo y el laboral. Su originalidad reside en haber considerado una serie de inputs no escolares y unos outputs basados en el punto de vista de la demanda, tal y como se propone desde el ámbito laboral. 


\section{Los resultados en formación profesional y su operativización}

Si bien es cierto que los resultados de los procesos de enseñanza-aprendizaje son múltiples y variados, sí que podríamos estar de acuerdo en que responden a las demandas sociales en un momento sociohistórico determinado, por lo menos en lo que respecta a la educación formal. En este sentido, el clásico debate sobre si formar para el trabajo o para el ejercicio de una ciudadanía activa y democrática pertenece más al ámbito ideológico que a las demandas reales de las familias (Fernández, 2016b), si bien es verdad que es imposible una cosa sin la otra. No obstante, la elección de ciclos formativos de formación profesional se suele realizar en base a su salida profesional (Rego-Agraso y Rial-Sánchez, 2017).

De un modo u otro, el sistema de formación profesional es el encargado de capacitar para el ejercicio de las actividades profesionales, una finalidad tradicionalmente, destinada a las clases populares como un itinerario de segunda. Así, no pasaría a formar parte del sistema educativo ordinario hasta la Ley General de Educación de 1970 en un esfuerzo por su legitimación como una opción profesionalizadora con entidad propia. De hecho, no ha comenzado a ocupar un papel relevante en las agendas de las políticas nacionales y europeas hasta hace relativamente poco (Jiménez, et al., 2015). No obstante, esta incorporación no fue plena, sino que estuvo caracterizada por la división de su oferta en el ámbito educativo y laboral, sin una articulación clara y respondiendo a dos tradiciones pedagógicas y evaluativas distintas: la primera anclada en una cultura academicista y la segunda, más cercana a las demandas de los mercados de trabajo.

No sería hasta la finalización del proceso de modernización de la formación profesional con la publicación en 2002 de la Ley de las Cualificaciones y la Formación Profesional cuando se puede hablar de un verdadero sistema integrado de formación profesional capaz de interconectar ambos escenarios entre sí. De este modo, la actual estructura del sistema de formación profesional en España se configura en torno al Sistema Nacional de Cualificaciones y Formación Profesional (SNCP) como conjunto de instrumentos y acciones capaces de promover y desarrollar la integración de las distintas ofertas de formación profesional mediante el Catálogo de Nacional de Cualificaciones Profesionales (CNCP) bajo los conceptos de cualificación y competencia profesional (Ley 5/2002 y Real Decreto 1128/2003).

Aunque se haya avanzado en la convergencia e integración de ambas ofertas, su división se ha mantenido. Dentro del ámbito educativo (Ley Orgánica 2/2006; Real Decreto 1147/2011) encontramos la formación profesional inicial o reglada (ciclos de formación profesional básica, de grado medio y de grado superior) y, en el laboral (Ley 30/2015) la formación programada por las empresas, la oferta formativa para trabajadores ocupados y desempleados, así como otras iniciativas de formación profesional para el empleo. Ambas ofertas coinciden en lo básico, en la inserción sociolaboral de sus egresados, si bien desde el ámbito laboral se hace más énfasis en la necesidad de responder a las demandas propias del tejido empresarial y económico. 
Tabla 1

Sintesis comparativa de las de finalidades de la Formación Profesional en los ámbitos educativo y laboral

\section{FORMACIÓN PROFESIONAL DEL SISTEMA EDUCATIVO (ÁMBITO EDUCATIVO)}

1. Preparar al alumnado (o cualificar a las personas) para el desempeño de una actividad en un campo profesional específico.

2. Contribuir al desarrollo económico del país.

3. Facilitar su adaptación a las modificaciones laborales que pueden producirse a lo largo de su vida.

4. Contribuir a su desarrollo personal.

5. Ejercicio de una ciudadanía democrática que permita la inclusión y cohesión social.

6. Aprendizaje a lo largo de la vida.

\section{FORMACIÓN PROFESIONAL PARA EL EMPLEO (ÁMBITO LABORAL)}

1. Mejora de las competencias profesionales y de los itinerarios de empleo y formación atendiendo a los requerimientos del mercado de trabajo y a las necesidades de las empresas. Reconocimiento y acreditación de las competencias profesionales adquiridas en ámbitos formales, no formales e informales (mediante la experiencia laboral.

2. Contribución a la mejora de la productividad y competitividad de las empresas.

3. Fomento del uso de las tecnologías de la información y la comunicación disminuyendo la brecha digital y garantizando la accesibilidad.

4. Desarrollo profesional y personal.

5. Mejora de la empleabilidad de los trabajadores, especialmente los de mayor dificultad para el mantenimiento del empleo o de inserción laboral.

6. Formación a lo largo de la vida de los trabajadores desempleados y ocupados.

Elaboración propia.

La incorporación del discurso de las competencias en el seno de las políticas educativas nacionales y europeas puede entenderse en base a la necesidad de incorporar las demandas del mercado laboral al sistema educativo. El concepto surgió en los años 70-80 como respuesta al cambio de modelo productivo que condujo a las sociedades y economías del conocimiento, consolidándose la noción de «competencia profesional» en los 90. El discurso generado en torno a la competencia pone el énfasis en el sentido práctico del conocimiento como un nuevo mainstream que no solo ha calado en 
formación profesional, sino también en el resto de enseñanzas del sistema educativo español (Lorente, 2012). Aunque no existe consenso sobre su definición, siendo este el principal inconveniente para su uso y operativización, podemos apuntar, grosso modo, que se trata de aquel conjunto de conocimientos, procedimientos y actitudes combinados, desarrollados desde múltiples lugares y formas donde la experiencia juega un papel fundamental en su adquisición (Tejada y Ruiz, 2016).

No obstante, su incorporación en las políticas educativas nacionales e internacionales a través de la reforma de la educación superior, no ha estado exenta de críticas. Unas más centradas en su implementación, inciden en la indefinición del término o la dificultad de su aplicación, aunque no suponen un cuestionamiento total (Gimeno, 2011), mientras que otras lo culpabilizan de mercantilizar la educación y vaciar de contenido su currículo desprofesionalizando al profesorado (Fernández, et al., 2017).

\section{La medición de resultados en formación profesional}

El uso de las competencias en los procesos de enseñanza-aprendizaje es complejo, siendo la evaluación uno de los aspectos que más controversia suscita. Evaluar no consiste solo en medir el aprendizaje, sino en facilitarlo; pero hacerlo desde el punto de vista de las competencias es más complejo, si cabe, debido a la dificultad conceptual señalada. Así, la evaluación de competencias profesionales en educación superior requiere atender a distintas demandas: interdisciplinariedad, referencia a un perfil profesional determinado e integración en escenarios formativos y sociolaborales, atención al carácter multidimensional de la evaluación y recogida de evidencias en base a la triangulación instrumental (Tejada y Ruíz, 2016).

De este modo, se hace necesaria la implicación de distintos agentes evaluadores y la puesta en marcha de procesos más participativos y democráticos (Muñoz y Herrera, 2017) tales como la autoevaluación, heteroevaluación y coevaluación presentes en el espacio clave en que se adquieren, desarrollan y evidencian las competencias, en el puesto de trabajo: "Los agentes evaluadores a los que nos referimos hay que buscarles en el propio escenario de actuación profesional donde se activa, expresa y evidencia la competencia profesional." (Tejada y Ruiz, 2016, p. 33-34).

La implantación del discurso de las competencias y de su evaluación en formación profesional ha seguido lógicas distintas en función de la tradición de partida. Así, en el ámbito educativo, más allá del debate sobre si evaluar conocimientos disciplinares o competencias profesionales, lo que ha permanecido invariable es el protagonismo del profesorado como único agente evaluador, no dando cabida al tejido empresarial al que, supuestamente, responden las competencias profesionales que se intentan desarrollar. El sector empresarial colabora con el Instituto Nacional de las Cualificaciones (INCUAL) en el diseño de las distintas cualificaciones profesionales que articulan la oferta de títulos de formación profesional, pero, en cuanto a la evaluación del alumnado, su participación es escasa. Por ejemplo, en los denominados módulos de formación en centros de trabajo, de carácter obligatorio y común tanto para los ciclos formativos de grado medio como superior, si bien existe la figura del tutor profesional como colaborador del tutor académico del centro, su función evaluativa es residual en tanto la calificación de estos módulos se realiza en términos de apto o no apto, no contando para el cálculo de la nota media del 
expediente académico (Real Decreto 1147/2011). De este modo, podríamos decir que la función de la evaluación consiste en escalar el grado de adquisición de competencias profesionales en forma de calificación, y para ello, es el profesorado, amparado en su libertad de cátedra, el principal protagonista en decidir con qué evidencias contar y qué instrumentos evaluativos utilizar.

Por el contrario, la lógica en el ámbito laboral es bien distinta. Se basa en acreditar, o no, la realización de determinadas acciones formativas o la adquisición de competencias profesionales en lugar de traducir los resultados de la evaluación a una calificación final. En el primer caso, mediante la expedición de un «diploma acreditativo» que constata su "evaluación positiva" -o, en caso contrario, mediante un "certificado de asistencia» (Ley 30/2015)- y, en el segundo, con la obtención de un certificado de profesionalidad o la acreditación de una unidad de competencia (la unidad mínima acreditable por el sistema). De este modo, la evaluación queda relegada en un primer momento a una función acreditativa, certificando, o no, la presencia de un determinado número de competencias profesionales para, en última instancia, ocuparse de su principal objetivo la medición del impacto de los conocimientos adquiridos en términos de empleabilidad e inserción laboral (Ley 30/2015). Para ello, fundamenta su evaluación en los modelos de evaluación del impacto de la formación en las organizaciones (Kirkpatrick y Kirkpatrick, 2007; Pineda, 2000; Tejada y Ferrández, 2012) donde es el alumnado el principal agente evaluador del proceso de enseñanza-aprendizaje. Estos modelos se suelen basar, con pequeños matices, en cuatro o cinco niveles de evaluación, siendo los tres primeros los que atañen directamente al individuo y donde se mide el grado de satisfacción con la formación recibida, el aprendizaje alcanzado y su posibilidad de transferencia al puesto de trabajo. Un cuarto nivel se encargaría de medir su impacto en términos monetarios o no monetarios, y un quinto de su rentabilidad (Pulliam y Phillips, 2007). En este sentido, la Fundación Estatal para la Formación en el Empleo (FUNDAE), entidad colaboradora y de apoyo técnico, entre otras funciones, del SEPE (Servicio Público de Empleo Estatal) y del MEFP (Ministerio de Educación y Formación Profesional), en tanto responsable de realizar la evaluación del sistema utiliza, para asegurar la calidad de los procesos de enseñanza-aprendizaje, cuestionarios normalizados y adaptados a cada una de las iniciativas de formación propias de este ámbito, pero con unos ítems o indicadores comunes en correspondencia directa con los tres primeros niveles de evaluación señalados.

Aunque ambos modelos utilicen la competencia profesional como referente evaluativo, los indicadores utilizados para medir su grado de adquisición varían en gran medida en función de su lógica y del protagonismo otorgado a un agente evaluativo u otro. Mientras la calificación responde más a una lógica academicista, los indicadores de impacto, por el contrario, rinden cuentas en el ámbito laboral al que, por otro lado, se dirige la finalidad de estas enseñanzas. De una $u$ otra forma, ambos se refieren a la adquisición de competencias profesionales, si bien, más allá de su tipología, lo crucial para establecer su valor es la calidad del proceso evaluativo seguido en función del grado de atención prestado a los retos propuestos por Tejada y Ruíz (2016) para la evaluación de competencias profesionales en educación superior.

Sería razonable pensar que la valoración de las competencias en forma de calificaciones por parte de los centros de enseñanza debiera de guardar una relación positiva con su valoración en forma de satisfacción, aprendizaje y transferencia por parte del 
alumnado, aunque alguna investigación al respecto apunte a lo contrario (Santos y Garrido, 2015). En caso de confirmarse esta hipótesis, podríamos decir que ambos indicadores serían congruentes.

\section{Personalidad eficaz y clase social subjetiva}

Ya desde el informe Coleman numerosas han sido las investigaciones que han apuntalado la tesis de que más recursos escolares (físicos, humanos, ratios, etc.) no generan necesariamente mayores o mejores resultados (Fernández, 2016a), de forma que son posiblemente otro tipo de inputs, los denominados «no escolares» (inteligencia, esfuerzo personal, estatus socioeconómico, etc.), los que tienen una mayor capacidad explicativa en este sentido. Así, las variables independientes utilizadas en este estudio tienen más que ver con aspectos contextuales y rasgos de personalidad del estudiante (variables de presagio) que con variables de proceso (forma o método).

Para fundamentar esta decisión, se realizaron 6 entrevistas no estructuradas de entre 20 y 45 minutos de duración aproximada a profesorado perteneciente a centros educativos de la provincia de Barcelona con un promedio de 24 años de experiencia, dos de ellos miembros de los centros en los que se ha basado la muestra utilizada. La entrevista giraba en torno a una única pregunta o foco de interés: ¿Cuáles son los principales factores de éxito y/o fracaso en los procesos de enseñanza-aprendizaje en formación profesional en términos tanto de rendimiento académico como de empleabilidad? El objetivo fue el de comprender, más que explicar, de la forma más abierta posible el discurso de estos/as expertos del tema en base a su trayectoria profesional.

$\mathrm{Su}$ principal conclusión acerca los factores determinantes del éxito o fracaso del alumnado, en términos de rendimiento académico, empleabilidad e inserción sociolaboral (es decir, de adquisición de competencias profesionales), apunta tanto al grado de adaptación de la formación profesional a los requisitos del mercado laboral, como sugieren otras investigaciones (Lorente, 2016), como a la presencia de determinadas características tales como la motivación y la actitud e implicación personal en el proceso de enseñanza-aprendizaje (es decir inputs «no escolares»). En este sentido, se ha utilizado el constructo «personalidad eficaz» en tanto recoge la mayoría de estas características, además de haber sido considerado en investigaciones similares (Santos y Garrido, 2015). Asimismo, se ha desarrollado en contextos empresariales estando relacionado con la eficacia académica, vocacional, social y en el ejercicio profesional (Martín del Buey, et al., 2008). Su versión original ha sido adaptada al contexto español de formación profesional, concretamente para las enseñanzas de grado superior, quedando compuesto por seis dimensiones que incorporaremos en nuestro análisis como variables independientes. Se define del siguiente modo:

Una persona eficaz es un ser vivo con conocimiento y estima de sí mismo (autoconcepto y autoestima) en proceso de maduración constante (en cualquier estado de su evolución) con capacidad (inteligencia) para lograr (eficacia) lo que desea (motivación) y espera (expectativa) empleando para ello los mejores medios (entrenamiento) posibles (eficiencia), controlando las causas (atribución de causalidad) de su consecución (éxito o fracaso), afrontando para ello las dificultades personales, circunstanciales y sociales (afrontamiento de problemas) que se presenten, tomando 
las decisiones adecuadas sin detrimento de sus buenas relaciones con los demás (empatía y comunicación) ni renuncia en ellas de sus aspiraciones personales justas (asertividad). (Martín del Buey, et al., 2015: 53)

Por otro lado, la investigación aporta bastantes evidencias como para pensar que estos inputs «no escolares» se encuentran a su vez relacionados con el entorno sociocultural y socioeconómico del alumnado (Torrents, et al., 2018). Así, el éxito o fracaso en los procesos de enseñanza-aprendizaje muchas veces se fragua en instancias distintas a las académicas teniendo que ver con variables relacionadas con el origen, o las desigualdades territoriales (Puelles, 2016) y, con la identidad de clase (Manstead, 2018).

La clase social es un concepto multidimensional y multideterminado, lo que dificulta su asunción unánime por parte de las distintas disciplinas académicas. Existen variedad de posturas acerca de su escasa relevancia e influencia en la configuración de los fenómenos sociales y/o psicológicos, si bien, son más numerosas las evidencias que sostienen su importancia. Su medición suele hacerse de acuerdo a indicadores objetivos tales como la renta, el nivel educativo o el prestigio ocupacional y subjetivos en base al auto-posicionamiento en un determinado lugar de la jerarquía social en comparación con los demás (Sautu, et al., 2020). No obstante, la denominada como «clase social subjetiva», ha demostrado ser más relevante incluso que la objetiva a la hora de afectar a ciertos parámetros psicosociales como la autoeficacia (Quiroga-Garza, et al., 2018) el bienestar subjetivo (Tan, et al., 2020) y la salud (Navarro-Carrillo, et al. 2018) o las relaciones interpersonales (Bjornsdottir, et al., 2017). De este modo, además de por su potencial explicativo, la utilizamos en este estudio en contraposición a la objetiva por su posibilidad de entrenamiento, transformación y mejora, hecho que nos parece crucial desde un punto de vista educativo y que, además, comparte con la «personalidad eficaz».

\section{Método}

\section{Objetivos}

El objetivo principal de esta investigación es tanto analizar la influencia de determinadas variables explicativas (inputs "no escolares") en los resultados educativos (variables dependientes) que se tienen en cuenta desde el modelo evaluativo utilizado en la formación profesional en el ámbito laboral (al considerarlo más pertinente para sus objetivos), como comprobar el grado de congruencia existente entre los indicadores utilizados por los dos modelos evaluativos utilizados en formación profesional.

Para ello hemos adoptado un enfoque econométrico basado en un modelo de análisis logit ordenado (regresión logística ordenada o modelo de probabilidades proporcionales). Se trata, en última instancia, de la utilización de una función de producción educativa - outputs educativos $=f$ (inputs educativos) - en tanto lo que nos interesa conocer es el grado de afectación de ciertos inputs a los outputs establecidos desde el punto de vista de la demanda, es decir: los que se están utilizando en la formación profesional en el ámbito laboral por las razones aducidas en el apartado anterior. Este modelo de probabilidades nos permite predecir un resultado determinado (outputs) en función de la respuesta a otras preguntas (inputs no escolares). Por otro lado, al tratarse de un estudio de carácter exploratorio hemos optado por un diseño transversal no experimental. 
Tabla 2

Variables dependientes y explicativas utilizadas

\section{VARIABLES DEPENDIENTES (Outputs)}

PRL (Satisfacción general con el módulo)

PRM (Satisfacción con el grado de aprendizaje adquirido)

PRN (Grado de utilidad de lo aprendido)

\begin{tabular}{ll}
\hline \multicolumn{2}{l}{ VARIABLES EXPLICATIVAS (Inputs) } \\
\hline VARIABLES INDEPENDIENTES & VARIABLES DE CONTROL \\
\hline AS (Autoconcepto social) & PRC (Género) \\
AR (Autoconcepto académico) & PRG (Años de experiencia laboral) \\
AU (Autoestima) & ADF (Actividades físicas y deportivas) \\
EE (Expectativa de éxito) & EOC (Edificación y obra civil) \\
AP (Afrontamiento de problemas) & IC (Informática y comunicaciones) \\
PRH (Clase social subjetiva) & IM (Instalación y mantenimiento) \\
PRK (Nota media del primer curso) & S (Sanidad) \\
& TMV (Transporte y mantenimiento de vehículos) \\
& IS (Imagen y sonido)
\end{tabular}

Elaboración propia.

\section{Población y Muestra}

Utilizamos una muestra no probabilística, no aleatorizada, por conveniencia de 374 estudiantes (59\% hombres y $41 \%$ mujeres). Al tratarse de un estudio exploratorio, hemos utilizado un procedimiento de muestreo casual o incidental donde se ha priorizado la facilidad de acceso toda vez que nuestra pretensión no ha sido la de establecer conclusiones generalizables para toda la población, sino la de indagar en la problemática presentada identificando algunas conclusiones a modo de hipótesis en las que fundamentar posteriores estudios. La población de referencia ha sido el alumnado matriculado en segundo curso de distintos Ciclos Formativos de Grado Superior durante el curso académico 2018-19 pertenecientes a cuatro centros educativos próximos geográficamente en la provincia de Barcelona (Cataluña-España). Tres de ellos pertenecen al municipio de Tarrasa y un cuarto a Sabadell. 
Tabla 3

Población y error muestral

\begin{tabular}{|c|c|c|c|c|}
\hline \multirow[t]{2}{*}{ Zona } & \multirow[t]{2}{*}{ Población } & \multicolumn{3}{|c|}{$\begin{array}{l}\text { Error muestral máximo (muestras finitas) por } \\
\text { nivel de confianza }\end{array}$} \\
\hline & & $95 \%$ & $97 \%$ & $99 \%$ \\
\hline Cataluña & 29.603 & $5.04 \%$ & $5.57 \%$ & $6.63 \%$ \\
\hline $\begin{array}{l}\text { Total } 4 \text { cen- } \\
\text { tros }\end{array}$ & 904 & $3.88 \%$ & $4.3 \%$ & $5.11 \%$ \\
\hline
\end{tabular}

Elaboración propia.

\section{Instrumento}

Se administró un cuestionario informatizado con diferentes tipos de respuesta compuesto por los siguientes elementos:

a) Datos generales: familia profesional a la que pertenece el ciclo, género y años de experiencia laboral. Se utilizaron respuestas cerradas con diferentes alternativas.

b) Grado de satisfacción, aprendizaje y transferencia de la formación recibida. Las respuestas se ordenaron mediante una escala discreta ordinal entre el 1 (Muy deficiente) y el 10 (Excelente).

c) Nota media del primer curso. Se utilizó una respuesta abierta.

d) Cuestionario Personalidad Eficaz-Formación Profesional (CPE-FP), el cual presenta una fiabilidad alfa de Cronbach de .84, estando compuesto por 24 ítems correspondientes a 6 dimensiones: autoestima (AU), autoconcepto académico (AA), expectativa de éxito (EE), capacidad resolutiva (CR), afrontamiento de problemas (AP) y autoconcepto social (AS), (Martín del Buey, et al., 2008). Se trata de un instrumento de medida del constructo "Personalidad eficaz", adaptado y validado para el alumnado español de los Ciclos Formativos de Grado Superior en Formación Profesional. Se utilizó una respuesta tipo Likert con 5 opciones: nunca, pocas veces, alguna vez, muchas veces, siempre.

e) Escala MacArthur de estatus socioeconómico subjetivo (Adler y Stewart, 2007). Las respuestas se ordenaron mediante una escala ordinal entre el 1 (Menor estatus social) y el 10 (Mayor estatus social) simulando una escalera con 10 escalones que representan la jerarquía social en la que debe de situarse el alumnado en comparación con el resto de las familias de su entorno.

Aunque el grosso del cuestionario corresponde a ítems pertenecientes a cuestionarios ya validados y adaptados, la versión final del mismo fue administrada a modo de pilotaje a 31 alumnos/as sin que hubiese que hacer modificaciones significativas.

\section{Procedimiento de recogida y análisis de datos}

El carácter exploratorio del presente estudio ha determinado en gran medida el procedimiento de recogida de datos. Nuestra pretensión de bosquejar hipótesis en las 
que fundamentar posteriores estudios ha marcado este proceso de recogida de información. De este modo, los criterios de selección de los centros para la recogida de la muestra han tenido más que ver con la facilidad de acceso al campo, de acuerdo con dicha pretensión exploratoria.

Así, dicho acceso fue facilitado tanto por algunos de los expertos entrevistados como por la cercanía profesional de uno de los autores del presente estudio. De este modo, en primer lugar, uno de los centros se ofreció a colaborar en la administración de los primeros 31 cuestionarios como decimos, a modo de pilotaje, y el resto colaboró en todo momento en la recogida de la muestra utilizada con alumnado perteneciente a las 8 familias profesionales consideradas.

La calidad de la muestra se ha asegurado con el desplazamiento de uno de los investigadores a cada uno de los centros, ocupándose de garantizar -en colaboración con la dirección y el profesorado- tanto la correcta comprensión del protocolo de aplicación del cuestionario, como la consecución de un adecuado clima de concentración durante su desarrollo. Del mismo modo, se informó del carácter anónimo del cuestionario.

Dado que las tres variables dependientes de interés se han medido utilizando una escala discreta ordinal, se ha usado un modelo econométrico logit ordenado. Un análisis multinivel no ha procedido en este caso al no tener en torno a 20 grupos en el nivel 2 (familias profesionales) y nivel 3 (centros). Se probó en cualquier caso en Stata y, por ejemplo, no estimaba las varianzas del tercer nivel. Así, para tener en cuenta las diferencias entre familias profesionales se ha optado por crear 8 grupos de dummies, y para tener en cuenta que los estudiantes están en cuatro grupos o centros educativos, se ha usado la opción clúster de Stata. Esta opción nos corrige los errores estándar, proporcionando errores estándar robustos, por lo que estamos seguros de los p-valores y de la significatividad de los coeficientes estimados. Además, se ha tenido en cuenta que los estudiantes están en clúster (pertenecen a 4 centros distintos).

\section{Resultados}

\section{Satisfacción general (PRL)}

Tabla 4

Relación de las variables de interés con la satisfacción general

\begin{tabular}{|c|c|c|c|c|}
\hline & \multicolumn{3}{|c|}{ Robust } & \multirow[b]{2}{*}{$P>z$} \\
\hline & Coef. & Std. Err. & $\mathbf{z}$ & \\
\hline Autoconcepto Social (AS) & 0.1134 & 0.1285 & 0.8800 & 0.3770 \\
\hline Autoconcepto Académico (AA) & 0.1459 & 0.1057 & 1.3800 & 0.1680 \\
\hline Capacidad Resolutiva (CR) & 0.1190 & 0.0582 & 2.0500 & 0.0410 \\
\hline Autoestima (AU) & -0.2445 & 0.0499 & -4.9000 & 0.0000 \\
\hline Expectativa de Éxito (EE) & 0.2606 & 0.0296 & 8.7900 & 0.0000 \\
\hline Afrontamiento de Problemas (AP) & -0.1628 & 0.0454 & -3.5800 & 0.0000 \\
\hline Clase Social Subjetiva (PRH) & 0.1309 & 0.0385 & 3.4000 & 0.0010 \\
\hline Nota media primer curso (PRK) & 0.0000 & 0.0000 & -0.9800 & 0.3270 \\
\hline
\end{tabular}

Elaboración propia. 
Con respecto a esta primera variable de interés, observamos cómo el autoconcepto social (AS) y académico (AA) no tienen una influencia significativa en la satisfacción general. Por el contrario, sí que influyen positivamente la capacidad resolutiva (CR) y el grado de expectativa de éxito (EE). Paradójicamente, una buena autoestima (AU) y capacidad de afrontamiento de problemas (AP), afectan negativamente a dicha satisfacción. Igualmente, aumenta a mayor grado de clase social subjetiva (PRH), mientras que la nota media del primer curso (PRK) no guarda ningún tipo de relación.

Tabla 5

Relación de las variables de control con la satisfacción general

\begin{tabular}{|c|c|c|c|c|}
\hline & & Robust & & \\
\hline & Coef. & Std. Err. & $\mathbf{z}$ & $\mathrm{P}>\mathrm{Z}$ \\
\hline Género (=1 mujer) (PRC) & -0.4570 & 0.2119 & -2.1600 & 0.0310 \\
\hline Años experiencia laboral (PRG) & -0.0049 & 0.0239 & -0.2000 & 0.8380 \\
\hline Famil & rofesiona & (PRA) & & \\
\hline Actividades físicas y deportivas (AFD) & 1.9097 & 0.1538 & 12.4200 & 0.0000 \\
\hline Edificación y obra civil (EOC) & 0.8280 & 0.1357 & 6.1000 & 0.0000 \\
\hline Hostelería y turismo (HT) & -0.4345 & 0.0737 & -5.9000 & 0.0000 \\
\hline Imagen y sonido (IS) & & & ef. & \\
\hline Informática y comunicaciones (IC) & -0.2440 & 0.1367 & -1.7800 & 0.0740 \\
\hline Instalación y mantenimiento (IM) & 1.2558 & 0.2522 & 4.9800 & 0.0000 \\
\hline Sanidad (S) & 0.1692 & 0.0338 & 5.0100 & 0.0000 \\
\hline Transporte y mantenimiento de vehíc & -04005 & 01032 & -3.8800 & 0.0000 \\
\hline
\end{tabular}

Elaboración propia.

Con respecto a las variables de control, la satisfacción disminuye en función del género femenino (PRG), siendo los años de experiencia laboral (PRG) irrelevantes. Por su parte, el alumnado de los ciclos formativos pertenecientes a las familias AFD, EOC, IM y $S$ son los que se sienten más satisfechos, al contrario de lo que ocurre con HT, TMV e IC, si bien la significatividad de este hecho para el caso de IC es algo menor.

\section{Percepción con el grado de aprendizaje (PRM)}

Tabla 6

Relación de las variables de interés con el grado de aprendizaje

\begin{tabular}{lllll}
\hline & \multicolumn{3}{c}{ Robust } & P $>\mathbf{z}$ \\
\hline Autoconcepto Social (AS) & Std. Err. & z & 1.9100 & 0.0560 \\
Autoconcepto Académico (AA) & 0.0895 & 0.0469 & 1.2400 & 0.2160 \\
\hline
\end{tabular}




\begin{tabular}{|c|c|c|c|c|}
\hline & Coef. & $\begin{array}{l}\text { Robust } \\
\text { Std. Err. }\end{array}$ & $\mathbf{z}$ & $\mathbf{P}>\mathbf{z}$ \\
\hline Capacidad Resolutiva (CR) & 0.0446 & 0.1076 & 0.4100 & 0.6780 \\
\hline Autoestima (AU) & -0.1084 & 0.0733 & -1.4800 & 0.1390 \\
\hline Expectativa de Éxito (EE) & 0.3842 & 0.0549 & 6.9900 & 0.0000 \\
\hline Afrontamiento de Problemas (AP) & -0.0721 & 0.0684 & -1.0500 & 0.2920 \\
\hline Clase Social Subjetiva (PRH) & 0.1507 & 0.0896 & 1.6800 & 0.0930 \\
\hline Nota media primer curso (PRK) & 0.0000 & 0.0000 & -0.5300 & 0.5960 \\
\hline
\end{tabular}

Elaboración propia.

Si atendemos al grado de aprendizaje percibido, la mayoría de las variables independientes tienen una influencia despreciable con la positiva excepción de la expectativa de éxito (EE) el autoconcepto social (AS) y la clase social subjetiva (PRH), si bien, estas dos últimas en menor medida. La nota media del primer curso (PRK) no guarda ningún tipo de relación.

Tabla 7

Relación de las variables de control con el grado de aprendizaje

\begin{tabular}{|c|c|c|c|c|}
\hline & Coef. & $\begin{array}{l}\text { Robust } \\
\text { Std. Err. }\end{array}$ & $\mathbf{z}$ & $\mathbf{P}>\mathbf{z}$ \\
\hline Género (=1 mujer) (PRC) & -0.4323 & 0.3814 & -1.1300 & 0.2570 \\
\hline Años experiencia laboral (PRG) & -0.0513 & 0.0278 & -1.8500 & 0.0650 \\
\hline \multicolumn{5}{|c|}{ Familias profesionales (PRA) } \\
\hline Actividades físicas y deportivas (AFD) & 0.8029 & 0.2003 & 4.0100 & 0.0000 \\
\hline Edificación y obra civil (EOC) & 0.9982 & 0.1333 & 7.4900 & 0.0000 \\
\hline Hostelería y turismo (HT) & -0.0628 & 0.0339 & -1.8500 & 0.0640 \\
\hline Imagen y sonido (IS) & \multicolumn{4}{|c|}{ Ref. } \\
\hline Informática y comunicaciones (IC) & -0.3379 & 0.1471 & -2.3000 & 0.0220 \\
\hline Instalación y mantenimiento (IM) & -0.2871 & 0.2558 & -1.1200 & 0.2620 \\
\hline Sanidad (S) & 0.7063 & 0.0339 & 20.8200 & 0.0000 \\
\hline $\begin{array}{l}\text { Transporte y mantenimiento de vehí } \\
\text { (TMV) }\end{array}$ & -0.8464 & 0.1825 & -4.6400 & 0.0000 \\
\hline
\end{tabular}

Elaboración propia.

Por otro lado, mientras que el género (PRC) no guarda relación alguna, una mayor experiencia laboral (PRG) genera menor satisfacción con lo aprendido. Por familias se encuentra más satisfecho el alumnado de AFD, EOC y $S$, mientras que en HT, IC y TMV lo están menos. 


\section{Percepción con la utilidad de lo aprendido (PRN)}

Tabla 8

Relación de las variables de interés con la utilidad de lo aprendido

\begin{tabular}{|c|c|c|c|c|}
\hline & Coef. & $\begin{array}{l}\text { Robust } \\
\text { Std. Err. }\end{array}$ & $\mathbf{z}$ & $\mathrm{P}>\mathrm{z}$ \\
\hline Autoconcepto Social (AS) & 0.0777 & 0.1442 & 0.5400 & 0.5900 \\
\hline Autoconcepto Académico (AA) & 0.2373 & 0.0767 & 3.0900 & 0.0020 \\
\hline Capacidad Resolutiva (CR) & 0.0572 & 0.0369 & 1.5500 & 0.1210 \\
\hline Autoestima (AU) & -0.1416 & 0.0592 & -2.3900 & 0.0170 \\
\hline Expectativa de Éxito (EE) & 0.3467 & 0.0848 & 4.0900 & 0.0000 \\
\hline Afrontamiento de Problemas (AP) & -0.0864 & 0.0215 & -4.0200 & 0.0000 \\
\hline Clase Social Subjetiva (PRH) & 0.1714 & 0.0927 & 1.8500 & 0.0650 \\
\hline Nota media primer curso (PRK) & 0.0000 & 0.0000 & -1.6400 & 0.1010 \\
\hline
\end{tabular}

Elaboración propia.

Por último, la transferencia al puesto de trabajo es mayor, cuanto mayor es el autoconcepto académico (AA) y la expectativa de éxito (EE), mientras que es menor a mayor autoestima (AU) y capacidad de afrontamiento de problemas (AP). El autoconcepto social (AS) y la capacidad resolutiva (CR) no son relevantes para esta variable. Por su parte, la nota media (PRK) sigue sin tener influencia, mientras que la clase social subjetiva (PRH) lo hace de forma positiva.

Tabla 9

Relación de las variables de control con la utilidad de lo aprendido

\begin{tabular}{lllll}
\hline & Coef. & $\begin{array}{l}\text { Robust } \\
\text { Std. Err. }\end{array}$ & $\mathbf{z}$ & P >z \\
\hline Género (=1 mujer) (PRC) & -0.3464 & 0.3101 & -1.1200 & 0.2640 \\
Años experiencia laboral (PRG) & -0.0550 & 0.0252 & -2.1800 & 0.0290 \\
Familias profesionales (PRA) & & & & \\
Actividades físicas y deportivas (AFD) & 0.5422 & 0.0900 & 6.0200 & 0.0000 \\
Edificación y obra civil (EOC) & 1.0951 & 0.2113 & 5.1800 & 0.0000 \\
Hostelería y turismo (HT) & 0.9085 & 0.0834 & 10.8900 & 0.0000 \\
Imagen y sonido (IS) & Ref. & & & \\
Informática y comunicaciones (IC) & -0.0195 & 0.1066 & -0.1800 & 0.8550 \\
Instalación y mantenimiento (IM) & 0.1398 & 0.1936 & 0.7200 & 0.4700 \\
Sanidad (S) & 0.6978 & 0.1277 & 5.4700 & 0.0000 \\
Transporte y mantenimiento de vehículos & & & & \\
(TMV) & -0.2937 & 0.2170 & -1.3500 & 0.1760 \\
\hline
\end{tabular}

Elaboración propia. 
Para finalizar, con respecto a la transferencia al puesto de trabajo vuelve a repetirse la anterior situación. El género (PRC) no influye, mientras que la experiencia laboral (PRG), lo hace negativamente, si bien en un grado de confianza sensiblemente menor. Por familias, le otorgan, de nuevo, mayor utilidad a lo aprendido AFD, EOC y $S$, a las que se suma HT, mientras que el resto no tienen ninguna influencia.

\section{Discusión y conclusiones}

La interpretación de los anteriores resultados nos permite identificar las siguientes conclusiones:

1. La expectativa de éxito y la motivación es uno de los principales predictores de las variables de impacto consideradas.

2. La clase social subjetiva influye positivamente en los resultados, si bien con mayor significatividad en cuanto a la satisfacción.

3. La familia profesional es una variable con gran potencial explicativo del grado de satisfacción, aprendizaje y transferencia percibidos.

4. No existe relación alguna entre las dos formas de evaluar el grado de adquisición de competencia profesionales, es decir, entre la calificación y los indicadores de impacto analizados.

Al tratarse de un estudio exploratorio, podríamos considerar estas conclusiones como hipótesis a confirmar en futuros estudios.

Con respecto a la primera cabe decir, como apuntábamos, que las seis dimensiones que componen el constructo «personalidad eficaz» en su adaptación para formación profesional, se corresponden con las cuatro principales que contempla su versión original: fortalezas del yo, demandas del yo, retos del yo y relaciones del yo. La «expectativa de éxito» y el «autoconcepto académico» se incluirían en la denominada "Demandas del Yo" que contempla todos los factores relacionados con la motivación de logro, las atribuciones o locus de control y las expectativas dando respuesta a las preguntas “¿Qué quiero?, ¿Qué expectativas tengo de conseguirlo?, y ¿De quién o de qué depende su consecución exitosa?" (Martín del Buey, et al., 2008: 224).

La «expectativa de éxito» ha sido la única dimensión relacionada positivamente con el grado de satisfacción, aprendizaje y transferencia, mientras que el «autoconcepto académico» mantiene dicha relación positiva solo en cuanto a la transferencia del conocimiento adquirido. En comparación con el resto de las dimensiones, son dichas "Demandas del Yo" las que determinan en mayor medida el grado de satisfacción, aprendizaje y transferencia con las competencias profesionales adquiridas, siendo la motivación uno de sus principales factores. Esta es fruto de tres momentos: presente (motivación estrictamente dicha), pasado (dimensión atribucional) y futuro (dimensión expectativa) (Martín del Buey, et al., 2000). La expectativa de éxito, por tanto, formaría parte de la motivación futura.

Son numerosos los estudios que relacionan la motivación y las expectativas positivas en educación superior, tanto del alumnado como de la comunidad educativa en general (Luzarraga, et al., 2017) con distintos outputs educativos, como la inserción laboral (Piqueras, et al. 2008) o el rendimiento académico en términos de calificaciones (Nájera, et 
al., 2020). Si bien sería necesario identificar qué tipo de motivación (intrínseca o extrínseca) y qué expectativas influyen positivamente en según qué tipo de resultados estemos considerando. En el ámbito de la formación profesional estos pueden ser múltiples y variados además de presentar matices que no se dan en otras etapas educativas.

No obstante, las características motivacionales del sujeto, así como las del resto que componen el constructo "personalidad eficaz», no han de ser entendidas como estáticas e invariables de modo que determinaran ineludiblemente el devenir del sujeto. Por el contrario, son susceptibles de entrenamiento a lo largo de todo el ciclo vital por lo que es posible mejorar su incidencia en el rendimiento académico (Martín del Buey, et al., 2015).

Efectivamente, atendiendo a nuestra segunda conclusión, las expectativas se encuentran condicionadas por la clase social (Elias y Daza, 2019). En este sentido, entendemos la clase social más como una posición determinada (consciente o inconscientemente) en base a una identidad y a un estilo de vida (Ball, 2003) que, en cuanto a un nivel de renta, tal y como propone el concepto de habitus propuesto por Bourdieu (1991) y su distinción entre capital económico, cultural y social (Bourdieu, 2001). Así, toda vez que la clase social subjetiva no es más que una determinada construcción en base a la percepción y comparación social, se convierte en una variable con un gran potencial de desarrollo desde los procesos de enseñanza aprendizaje. Se trata de un componente básico en la conformación de la propia subjetividad, la cual se lleva a cabo mediante distintos procesos de apropiación discursiva. Esto implica la importancia de trabajar desde los ámbitos educativos en la identificación y construcción de la identidad como sugieren algunos estudios (Subero y Esteban-Guitart, 2020).

En tercer lugar, constatamos una valoración distinta del grado de satisfacción, aprendizaje y transferencia en función de la familia profesional del ciclo, siendo unas veces positivo y otras negativo. En este sentido, otros estudios señalan la importancia de considerar esta variable en tanto es capaz de explicar outputs como la expectativa de empleo (Rego-Agraso y Rial-Sánchez, 2017), si bien en próximas investigaciones sería necesario profundizar en este hecho.

En cuarto y último lugar, quizá la conclusión más interesante del análisis sea el no haber encontrado, como se esperaba, relación alguna entre la calificación y la valoración del aprendizaje: la variable independiente "nota media del primer curso" (PRK) no afecta al grado de satisfacción, aprendizaje y transferencia con la formación realizada. Así, podemos confirmar las conclusiones de la investigación de Santos y Garrido (2015), si bien la investigación sobre las relaciones entre la formación profesional en los ámbitos educativo y, sobre todo, laboral, al respecto es escasa o inexistente (Echeverría y Martínez, 2021). Este hallazgo, tan relevante como anti-intuitivo, nos permite afirmar que, los distintos indicadores de valoración de competencias considerados en este estudio no guardan relación entre sí, o lo que es lo mismo, que los distintos agentes evaluadores llegan a conclusiones diferentes a la hora de valorar el grado de adquisición de la competencia profesional.

No obstante, el debate sobre qué se entiende por resultados educativos es amplio y complejo, por lo que la reducción que hemos realizado sobre ellos entendiéndolos en forma de valoración del alumnado y como calificación podría señalarse como excesivamente reduccionista. Somos conscientes de ello, si bien su utilización ha 
respondido a criterios prácticos que han permitido operativizar nuestro objeto de estudio, pero, sobre todo, a la práctica evaluativa institucional. Además, cuando se mide el rendimiento académico en forma de calificación, no estamos seguros de que se estén utilizando los mismos instrumentos, criterios y evidencias para todo el alumnado, ya que esto queda al arbitrio del profesorado.

Dada la escasa literatura académica en formación profesional a la que hacemos referencia (Echeverría y Martínez, 2021), sería necesario profundizar en este tema de modo que podamos comprender el supuesto desajuste entre la valoración del grado de adquisición de competencias profesionales realizado desde el punto de vista educativo y laboral. Igualmente, habría que indagar en la naturaleza y etiología de este hecho. De manera tentativa, podríamos apuntar a la existencia del distinto grado de atención prestado a los retos propuestos por Tejada y Ruíz (2016) apuntados al inicio para la evaluación de competencias profesionales en educación superior para los ámbitos educativo y laboral.

Para finalizar, creemos necesario dar un paso más en el proceso de integración de la formación profesional en sus dos ámbitos de desarrollo, más allá del establecimiento de un sistema de convalidaciones en torno a la unidad de competencia como elemento de intercambio. Por su especial relevancia tanto para el propio proceso de enseñanzaaprendizaje como para el aseguramiento del impacto de sus resultados finales, sería beneficioso iniciar un diálogo entre los agentes involucrados en la evaluación de competencias profesionales capaz de establecer puentes sobre la pertinencia y utilidad de los actuales modelos de evaluación para los fines que la ley establece, así como para pensar un nuevo modelo de colaboración entre los escenarios académicos y laborales en el que se desarrolle el aprendizaje desde la idiosincrasia propia del contexto (Choy, et al., 2018). En este sentido, es especialmente interesante la propuesta de generar un ecosistema de Investigación sobre la Formación Profesional en España (IFPE) realizada por Echeverría y Martínez (2021). La valoración del grado de competencias profesionales adquirido en cualquiera de sus formas es compleja y entendemos que se debiera de avanzar hacia procesos evaluativos mucho más participativos, sin perjuicio de minar la libertad de cátedra del profesorado.

\section{Referencias}

Adler, N. E. y Stewart, J. (2007). The MacArthur Scale of subjective social status. Psychosocial Research Notebook. https://www.macses.ucsf.edu/research/psychosocial/ subjective.php

Ball, S. J. (2003). Class Strategies and the Education Market. The Middle Classes and Social Advantage. Londres: Routledge Falmer.

Bjornsdottir, R.T., Alaei, R. y Rule, N.O. (2017). The Perceptive Proletarian: Subjective Social Class Predicts Interpersonal Accuracy. Journal of Nonverbal Behavior, 41, 185-201 (2017). https://doi.org/10.1007/s10919-016-0248-6

Bourdieu, P. (1991). El Sentido Práctico. Madrid: Taurus.

Bourdieu, P. (2001). Poder, Derecho y Clases Sociales. Bilbao: Desclée de Brouwer.

Choy, S., Wärvik, G. B. y Lindberg, V. (Eds.). (2018). Integration of Vocational Education and Training Experiences: Purposes, Practices and Principles. Dordrecht: Springer. 
Echeverría, B. y Martínez, P. (2021). Hacia un ecosistema de investigación sobre formación profesional en España. Revista de Investigación Educativa, 39(1), 249-264. https:// doi.org/10.6018/rie.424901

Elias, M. y Daza, L. (2019). Configuración y Reconfiguración de las Expectativas Educativas después de la Educación Obligatoria: Un Análisis Longitudinal. International Journal of Sociology of Education, 8(3), 206-235. https://doi.org/10.17583/rise.2019.4479

Fernández, C., García, O. y Galindo, E. (2017). Escuela o barbarie: entre el neoliberalismo salvaje y el delirio de la izquierda. Madrid: AKAL.

Fernández, M. (2016a). El informe Coleman: una lección de sociología y de política. Revista de la Asociación de Sociología de la Educación, 9(1), 37-45.

Fernández, M. (2016b). La educación en la encrucijada. Madrid: Fundación Santillana.

Gimeno, J. (Comp.) (2011). Educar por competencias ¿qué hay de nuevo? Madrid: Morata.

Jiménez, M., Luengo, J. y Sevilla, D. (2015) Las políticas y legislación españolas de formación profesional: las consecuencias de la aprobación de la Ley de Cualificaciones y de la Formación Profesional y de la Ley de Economía Sostenible. En A. Luzón, y M. Torres (Comp.) Las políticas de formación profesional en España y en Europa: perspectivas comparadas (pp. 255-278). Barcelona: Octaedro.

Kirkpatrick, D. y Kirkpatrick, J. (2007). Evaluación de acciones formativas. Los cuatro niveles. Barcelona: Gestión 2000.

Ley 30/2015, de 9 de septiembre, por la que se regula el Sistema de Formación Profesional para el empleo en el ámbito laboral (B.O.E. no 207 de 10 de septiembre)

Ley 5/2002, de 19 de junio, de las Cualificaciones y de la Formación Profesional (B.O.E. no 147 de 20 de junio)

Ley Orgánica 2/2006, de 3 de mayo, de Educación (B.O.E. nº106, de 04 de mayo) modificada por la Ley Orgánica 8/2013, de 9 de diciembre, para la mejora de la calidad educativa (B.O.E. no 295 de 10 de diciembre)

Lorente, R. (2012). La formación profesional según el enfoque de las competencias. La influencia del discurso europeo en España. Barcelona: Octaedro.

Lorente, R. (2016). Perspectivas del profesorado ante los retos y desafíos de la formación profesional. Revista Qurriculum, 29, 63-86.

Luzarraga, J.M., Nuñez, J.M. y Etxeberria, J. (2018). Análisis de las expectativas de los centros de Bachillerato de alta y baja eficacia escolar. Percepción de la Inspección Educativa. Revista Complutense de Educación, 29(4), 1075-1090. https://doi.org/10.5209/ RCED.54683

Manstead, A. S. (2018). The psychology of social class: How socioeconomic status impacts thought, feelings, and behaviour. British Journal of Social Psychology, 57(2), 267-291. https://doi.org/10.1111/bjso.12251

Martín del Buey, F., Granados, P., Martín, M. E., Juárez, A., García, A. y Álvarez, M. (2000). Desarrollo de la Personalidad Eficaz en Contextos Educativos. Marco Conceptual. Oviedo: Fmb.

Martín del Buey, F.A., Martín, M. E., Dapelo, B., Pizarro, P., Di Giusto, C., Castellano, S., Guerra, P. y Arnáiz, A. (2015). El constructo personalidad eficaz: últimos avances. Revista de Orientación Educacional, 29(55), 52-68. 
Martín del Buey, Fo A., Fernández Zapico, A., Martín Palacio, E., Dapelo Pellerano, B., Marcone Trigo, R., y Granados Urban, P. (2008). Cuestionario de personalidad eficaz para la formación profesional. Psicothema, 20(2), 224-228.

Muñoz, D. y Herrera, D. (2017). Los desafíos de la evaluación por competencias en el ámbito educativo. Educação e Pesquisa, 43(4), 1073-1086. https://doi.org/10.1590/ s1678-4634201706164230

Nájera, J., Salazar, M. L., Vacio, M.A. y Morales, S. (2020). Evaluación de la autoeficacia, expectativas y metas académicas asociadas al rendimiento escolar. Revista de Investigación Educativa, 38(2), 435-452. https://doi.org/10.6018/rie.350421

Navarro-Carrillo, G.; Valor-Segura, I. y Moya, M. (2018). Clase social y bienestar subjetivo: el rol mediador de la incertidumbre emocional. Behavioral Psychology / Psicología Conductual, 26(3), 457-472.

Pineda, P. (2000). Evaluación del impacto de la formación en las organizaciones. Educar, 27, 119-133.

Piqueras, R., Rodríguez, A. y Rueda, C. (2008). Expectativas y duración del desempleo. Revista de Psicología del Trabajo y de las Organizaciones, 24(2), 129-151.

Puelles, M. (2016). Reflexiones sobre cuarenta años de educación en España o la irresistible seducción de las leyes. Historia y Memoria de la Educación, 3, 15-44. https:// doi.org/10.5944/hme.3.2016.14760

Pulliam, P. y Phillips, J. (2007). Fundamentos del ROI. Barcelona: Gestión 2000.

Quiroga-Garza, A., García-Sánchez, E., Treviño, F.A. y Willis, G.B. (2018). Relación entre clase social subjetiva y autoeficacia: efecto de la comparación social. Anales de psicología, 34(2), 314-323. http://doi.org/10.6018/analesps.34.2.266611

Real Decreto 1128/2003, de 5 de septiembre, por el que se regula el Catálogo Nacional de Cualificaciones Profesionales (B.O.E. no 223 de 17 de septiembre)

Real Decreto 1147/2011, de 29 de julio, por el que se establece la ordenación general de la formación profesional del sistema educativo (B.O.E. no 182 de 30 de julio)

Rego-Agraso, L. y Rial-Sánchez, A. (2017). ¿Por qué elegir formación profesional? Satisfacción, motivaciones y expectativas del alumnado sobre el empleo y la formación. Revista Española de Orientación y Psicopedagogía, 28(3), 43-62.

Santos, M. V. y Garrido, M. J. (2015). Resultado del proceso educativo: El papel de los estilos de aprendizaje y la personalidad. Educación XX1, 18(2), 323-349. https://doi. org/10.5944/educxx1.14607

Sautu, R., Boniolo, P., Dalle, P. y Elbert, R. (Ed.) (2020) El análisis de clases sociales. Buenos Aires: CLACSO.

Subero, D. y Esteban-Guitart, M. (2020). Más allá del aprendizaje escolar: el rol de la subjetividad en el enfoque de los fondos de identidad. Teoría de la Educación. Revista Interuniversitaria, 32(1), 213-236. http://doi.org/10.14201/teri.20955

Tan, J. J. X., Kraus, M. W., Carpenter, N. C., y Adler, N. E. (2020). The association between objective and subjective socioeconomic status and subjective well-being: A meta-analytic review. Psychological Bulletin, 146(11), 970-1020. https://doi.org/10.1037/ bul0000258 
Tejada, J. y Ferrández, E. (2012). El impacto de la formación continua: claves y problemáticas. Revista Iberoamericana de Educación, 58(3), 1-14. https://doi.org/10.35362/ rie5831427

Tejada, J. y Ruiz, C. (2016). Evaluación de competencias profesionales en Educación Superior: Retos e implicaciones. Educación XX1, 19(1), 17-38. https://doi.org/10.5944/ educxx1.12175

Torrents, D., Merino, R., García, M. y Valls, O. (2018). El peso del origen social y del centro escolar en la desigualdad de resultados al final de la escuela obligatoria. Papers, 103(1), 29-50. https://doi.org/10.5565/rev/papers.2300

Fecha de recepción: 5 de noviembre de 2020.

Fecha de revisión: 21 de noviembre de 2020.

Fecha de aceptación: 11 de febrero de 2021. 